\title{
Mining the proteome associated with rheumatic and autoimmune diseases
}

Cristina Ruiz-Romero ${ }^{1 *}$, Maggie P. Y. Lam ${ }^{2}$, Peter Nilsson ${ }^{3}$, Patrik Önnerfjord ${ }^{4}$, Paul J. Utz $^{5}$, Jennifer E. Van Eyk ${ }^{6}$,Vidya Venkatraman ${ }^{6}$, Justyna Fert-Bober ${ }^{6}$, Fiona E. Watt ${ }^{7}$, Francisco J. Blanco ${ }^{8 *}$

${ }^{1}$ Grupo de Investigación de Reumatología (GIR), Unidad de Proteómica, INIBIC - Complejo Hospitalario Universitario de A Coruña, SERGAS, Universidad de A Coruña, A Coruña, Spain

${ }^{2}$ Department of Medicine, Division of Cardiology, Consortium for Fibrosis Research and Translation, Anschutz Medical Campus, University of Colorado Denver, Aurora, Colorado 80045, United States

${ }^{3}$ Division of Affinity Proteomics, SciLifeLab, Department of Protein Science, KTH Royal Institute of Technology, Stockholm, Sweden

${ }^{4}$ Department of Clinical Sciences, Section for Rheumatology and Molecular Skeletal Biology, Lund University, Sweden

${ }^{5}$ Division of Immunology and Rheumatology, Stanford University School of Medicine; Palo Alto, California, United States

${ }^{6}$ Department of Medicine and The Heart Institute, Cedars-Sinai Medical Center, Los Angeles, California, United States

7 Arthritis Research UK Centre for Osteoarthritis Pathogenesis, Kennedy Institute of Rheumatology, University of Oxford, Oxford, United Kingdom.

${ }^{8}$ Grupo de Investigación de Reumatología, INIBIC-Complejo Hospitalario Universitario de A Coruña, SERGAS, Departamento de Medicina Universidad de A Coruña, A Coruña, Spain

\section{Supporting Information Table of Contents}

Supplementary Figure S1. Functional networks built with the top 50 popular proteins in Psoriatic Arthritis (PsA), Ankylosing Spondylitis (AS), Systemic Sclerosis (SSc) and Sjögren's Syndrome $(\mathrm{SjS})$.

Supplementary Figure S2. Overlapping of the top 100 popular proteins associated with RAD and other HPP initiatives, using the two literature mining tools.

Supplementary Figure S3. Percentages of common proteins identified in the top 100 with the two literature mining tools employed in this work. 
Supplementary Table S1. Top 100 proteins in representative RADs, using the PubPular tool. Provided as an excel table.

Supplementary Table S2. Top 100 proteins in representative RADs, using the PURPOSE tool. Provided as an excel table.

Supplementary Table S3. Functional biological process-enrichment analysis of the top 50 most popular proteins in RADs. Provided as an excel table. These data are illustrated in Figure 3 and Supplementary Figure S1.

Supplementary Table S4. Overlapping of RAD-associated proteins with the top 100 popular proteins in other HPP initiatives. Data are illustrated in Supplementary Figure S2.

Supplementary Table S5. Overlapping between the top 100 proteins that were identified as associated with representative RADs using the two literature mining engines employed in this work.

Supplementary Table S6. Overlapping of the top 100 popular proteins identified in each of the 7 representative RADs that have been analyzed. Provided as an excel table. 
PsA

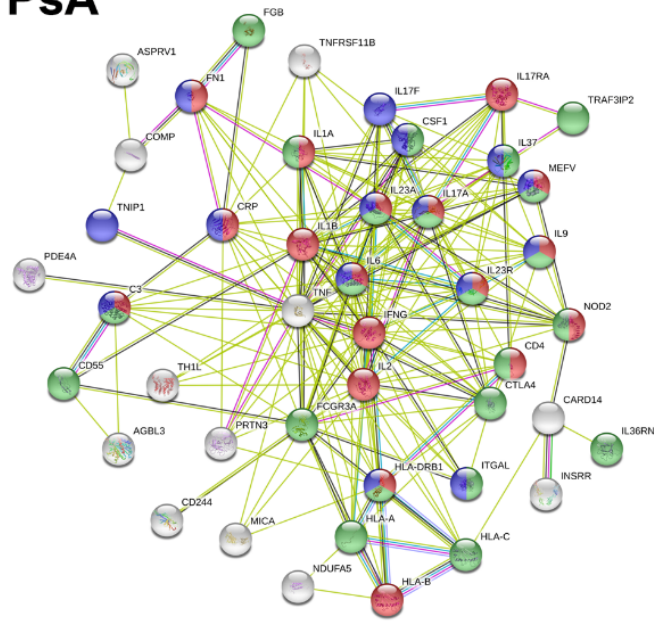

SSc

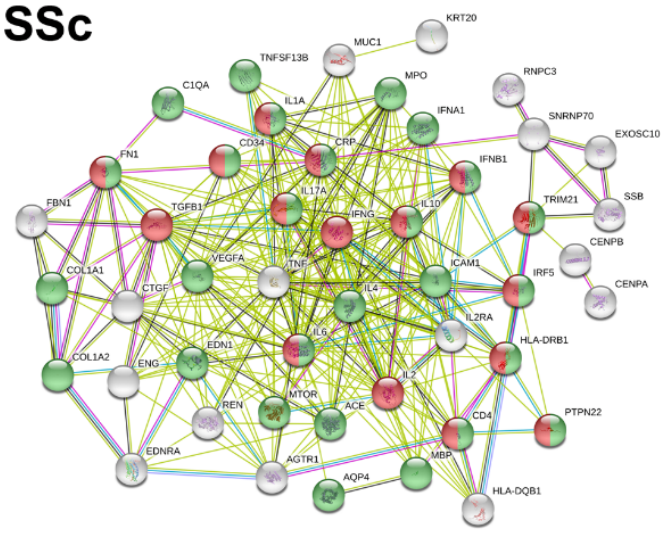

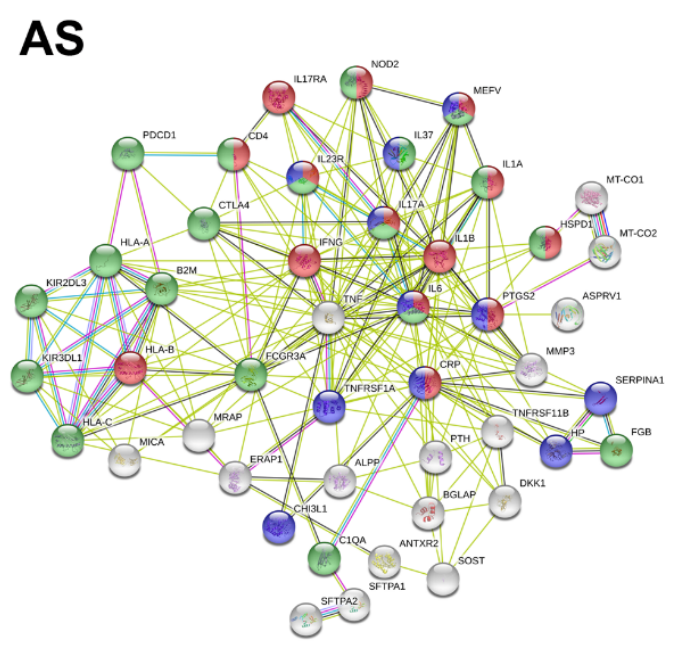

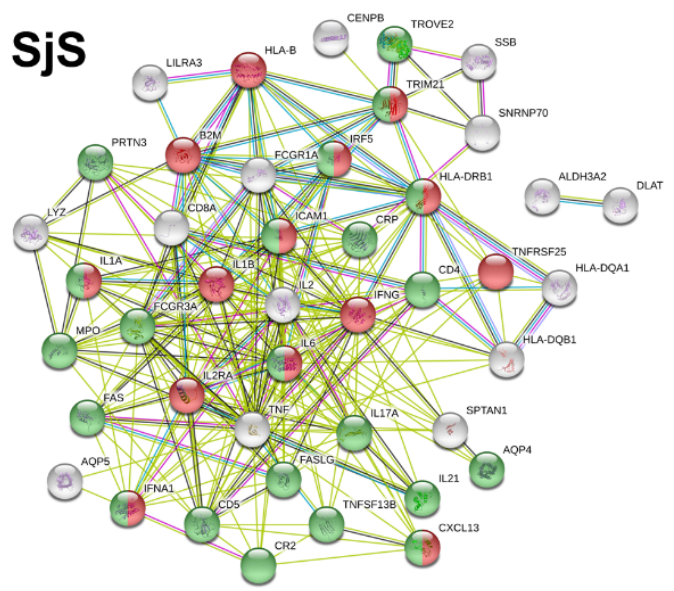

Supplementary Figure S1. Functional networks built with the top 50 popular proteins in Psoriatic Arthritis (PsA), Ankylosing Spondylitis (AS), Systemic Sclerosis (SSc) and Sjögren's Syndrome $(\mathrm{SjS})$. 


\section{PUBPULAR}

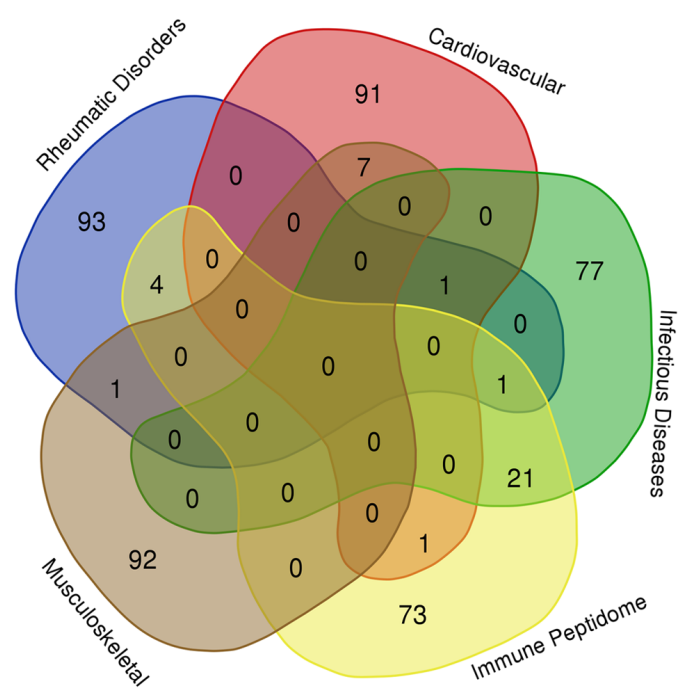

PURPOSE

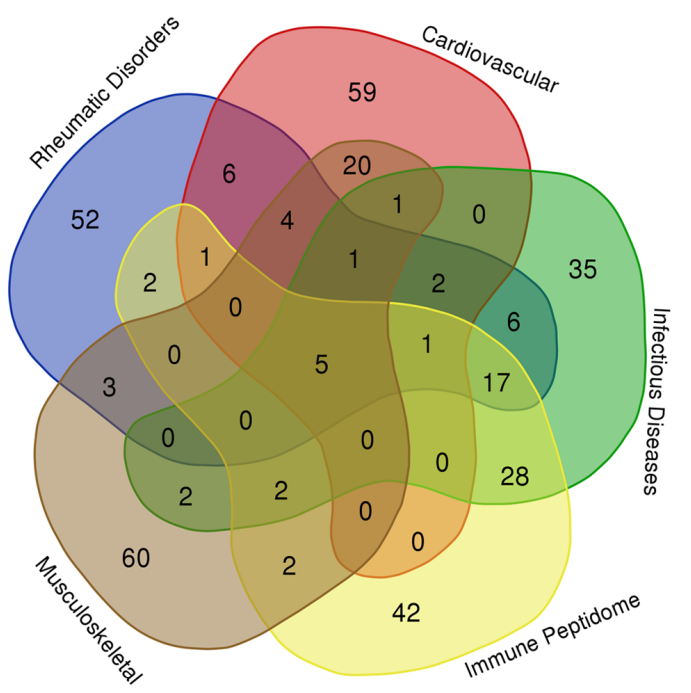

Supplementary Figure S2. Overlapping of the top 100 popular proteins associated with RAD and other HPP initiatives, using the two literature mining tools. The proteins in each group are enumerated in the Supplementary Table S5.

\section{Overlapping of the Top 100 proteins with PURPOSE}

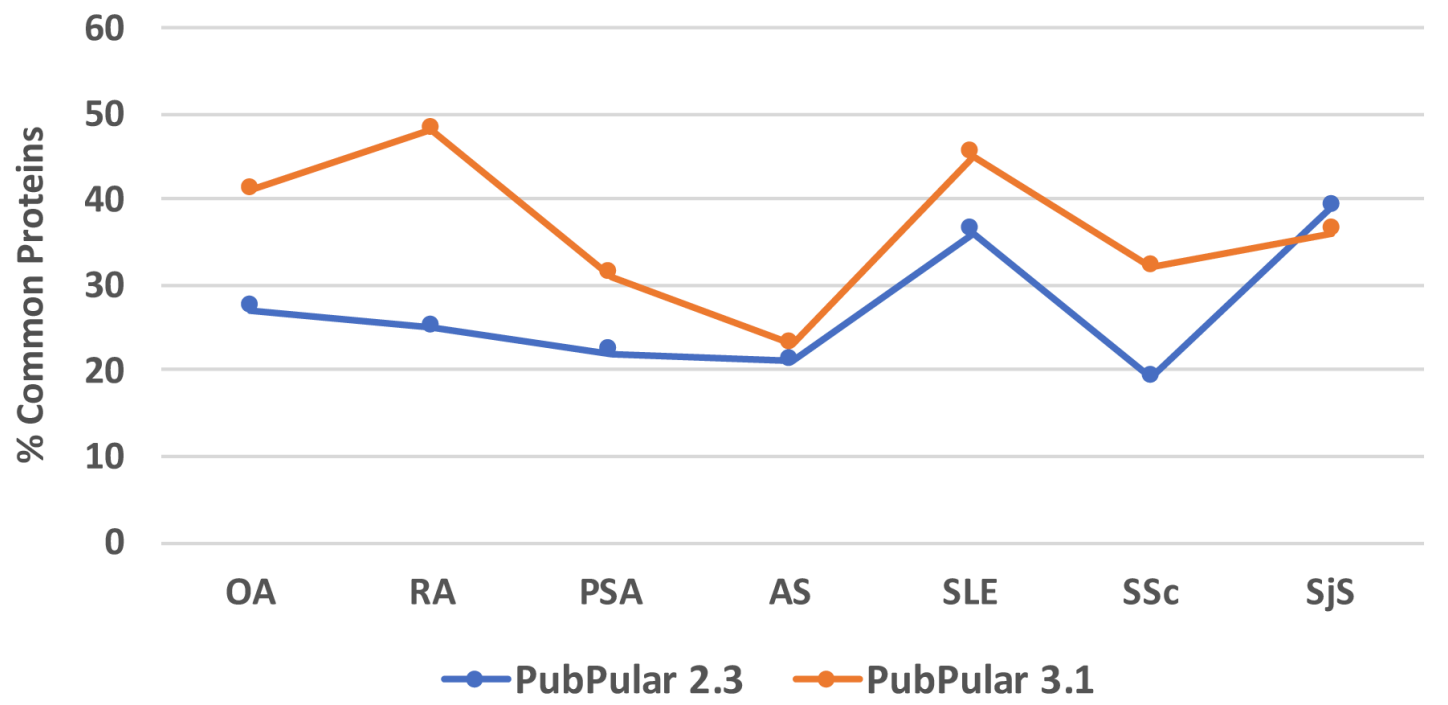

Supplementary Figure S3. Percentages of common proteins identified in the top 100 with the two literature mining tools employed in this work. 